\title{
Manufacturing Solution for Producing Cobalt-ferrite Films
}

\author{
Patrusheva T.N. ${ }^{1, *}$, Podorojnyak S.A. ${ }^{1}$, Polyakova K.P. ${ }^{2}$, Kirik S.D. ${ }^{3}$, Menshikov V.V. ${ }^{4}$, Levchenko S.I. ${ }^{4}$, \\ Patrushev V.V. ${ }^{3}$, Adrianova M.A. ${ }^{1}$, Gerasimova G.E. ${ }^{1}$ \\ ${ }^{1}$ Siberian Federal University, Krasnoyarsk,660044, Russia \\ ${ }^{2}$ L.V. Kirensky Institute of Physics SB RAS, Krasnoyarsk, Russia \\ ${ }^{3}$ Institute of Chemistry and Chemical Technology SB RAS, Krasnoyarsk, Russia \\ ${ }^{4}$ Siberian State Technological University, Krasnoyarsk, Russia \\ *Corresponding Author: pat55@mail.com
}

Copyright $@ 2014$ Horizon Research Publishing All rights reserved.

\begin{abstract}
An extraction-pyrolytic technique and its usage for obtaining pure products from secondary manufacturing solutions are considered in this paper. Nanostructure ferrite thin films were prepared using the extraction-pyrolytic technique. It was shown that the extraction from modeling liquid of waste solution allows obtaining the pure single phase product. $\mathrm{Fe}_{2} \mathrm{CoO}_{4}$ magnetic films with grain sizes $18-20 \mathrm{~nm}$ were obtained by cycling pyrolysis of wet films deposited by the spin coating technique.
\end{abstract}

Keywords Composite Oxides, Wet Technique, Extraction, Nanosize Materials, Homogeneity

\section{Introduction}

Currently great attention is given to the synthesis of nanosize materials. It is known that the presence of impurities influences greatly the characteristics of nanostructure materials, therefore expensive high-purity substances are used in their synthesis. The essential requirements for producing high quality functional materials are the achievement of chemical and phase composition homogeneity and set elements proportion. These requirements have been taken into account under the development of an extraction-pyrolysis technique for preparation of pure and homogeneous materials.

The usage of conventional techniques for acquiring composite oxide materials such as electron-beam evaporation, chemical vapor deposition, laser ablation techniques and the sol-gel method provide different properties of materials. Among the various techniques the wet chemical method such as sol-gel [1] and spray pyrolysis [2] provide the soft condition of composite oxides synthesis, These technologies would allow preparing the composite oxides under lower temperature and shorter time period for synthesis. They would offer an easy, scalable and cost-effective method for the preparation of films with ultra-fine particles [3].

With respect to polycrystalline films the properties depend on the grain boundary effects and orientation of the grains. The impurities incorporated during the growth are localized as usual at the grain boundaries and modulate the physical properties of materials. Therefore, the development of new a technology for the synthesis of composite oxide materials without impurities and under soft deposition conditions would be meaningful.

\section{Materials and Methods}

Polycrystalline or amorphous ferrite films are of commercial interest due to their low production cost. In this respect, the technology for synthesizing polycrystalline cobalt ferrite films with high perpendicular magnetic anisotropy and high magnetooptical effects emerged as a more important point [4]. The surface roughness of the polycrystalline films would be significantly larger than those of nanocrystalline materials. Therefore, syntheses of fine-grain ferrite films at lower temperature are the subject of the current research. Structural anomalies and disordering associated with the magnetic films having such small grains would influence the magnetic properties significantly.

For the synthesis of polycrystalline ferrite films we propose the extraction-pyrolysis method which consists in extraction of meaningful components from aqueous solutions of inorganic salts with the following mixture of the liquid extracts in the set ratio and deposition of solution on the substrate and thermal treatment [5].

The inorganic salts usually contain impurities. but the extraction process allows the cleaning of the impurities from the components. The concentrations of metals in extracts have been checked by the atomic absorption method. The following mixing of the extracts provides the exact 
component ratio in the final product.

The solution of monocarboxylic acids with number of carbon atoms $\mathrm{C}_{5}-\mathrm{C}_{9}$ (secondary solution from oil treatment, SPO Kaustic, Sterlitamak, Russia) used as extractant (VIC) for synthesis of $\mathrm{Co}$ (II) and $\mathrm{Fe}$ (III) extracts from inorganic solutions of $\mathrm{CoCl}_{2}$ and $\mathrm{FeCl}_{3}$ (OOO Reactiv, Krasnoyarsk, Russia). As monocarboxylic acids have low values of acidic dissociation, so the Fe and Co extraction was performed by the adding of sodium hydroxide solution to the aqueous solutions of metal salts. In an extraction system with monocarboxylic acids the additional interactions are presented, in particular, the formation of complicated carboxylates or three-kernel compounds of Fe (III) with $\mathrm{Co}(\mathrm{II})$. The possibility of interaction between $\mathrm{Fe}$ and $\mathrm{Co}$ carboxylates is promoted by a singlephase product creation at the pyrolysis stage of extraction.

As the result of 2-3 steps of extraction the carboxylates of metals without impurities were created. The previously calculated concentration of metal carboxylates underwent control by the atomic absorption spectroscopy (AAS). The solutions of carboxylates were mixed in the required proportions and used for films deposition and acquisition of ultra fine powders. There is no subdivided crystallization in organic solutions as in the aqua solutions.

The deposition of extracts on the different substrates was performed by immersion or spin coating techniques. The organic extracts can easily moisten any substrates due to the low values of the surface tension, and the following heat treatment improves film adhesion. The films obtained by this method have a lot of crystallization centers which promoted the formation of ultra fine structures in the solid phase.

Thermal decomposition (DTA) investigations were performed with Q-1000 device. X-ray diffraction (XRD) was performed in orders to determine the phase assemblage. The gas-chromatography (GC) method was employed for identifying the gaseous products from organic phase destruction. Analysis of carbon in the final product was performed with the C-N-elemental analyzer. Chemical composition and thickness of the films were determined by $\mathrm{X}$-ray fluorescence analysis. Magnetic and magneto-optical parameters were evaluated by using of torque meter and magneto-optical methods in the wavelength range $0.3-0.9$ microns in a field up to $1280 \mathrm{kA} / \mathrm{m}$. In particular, saturation magnetization and perpendicular magnetic anisotropy constant are measured by using the gyrating magnetic moment method. The values of the coercive force were obtained from magnetooptic closed hysteresis loops. Magnetooptical values were calculated from the Faraday rotation and absorption spectra.

At the creation of functional materials the crucial role is in providing the highest physical parameters with respect to the purity of the initial substances. The increase in the purity of the components is accompanied with by a concomitant complexity of treatment technologies, which is reflected in their cost. Thus, according to [6] the cost of powders with normal size distribution increases 4-20 times with respect to the decrease of the content of impurities. The usage of the extraction-pyrolytic method augments the profound removal of impurities at the extraction and can significantly reduce the cost of obtaining materials with the desired properties.

\section{Result and Discussion}

\subsection{Manufacturing Solution for Producing Cobalt-ferrite Films}

The possibility of deep cleaning of cobalt and iron during the extraction stage was tested on a model sulfate-chloride solution corresponding to the composition of the manufacturing solution of cobalt production plant (Norilsk metallurgy). The stock solution includes $3.5 \mathrm{~g} / 1 \mathrm{Cu}, 3.0 \mathrm{~g} / 1 \mathrm{Fe}$, $65.0 \mathrm{~g} / \mathrm{l} \mathrm{Co}$. The technological scheme for obtaining of the iron and cobalt extracts is shown in Fig.1.

At the first stage the $\mathrm{Fe}$ and particularly $\mathrm{Cu}$ were extracted from the stock solution of metal salts by adding an equivalent amount of $4 \mathrm{M} \mathrm{NaOH}$ solution to the total content of iron and copper. The volume ratio of the phases was $\operatorname{Org}: \mathrm{W}=1: 5$.

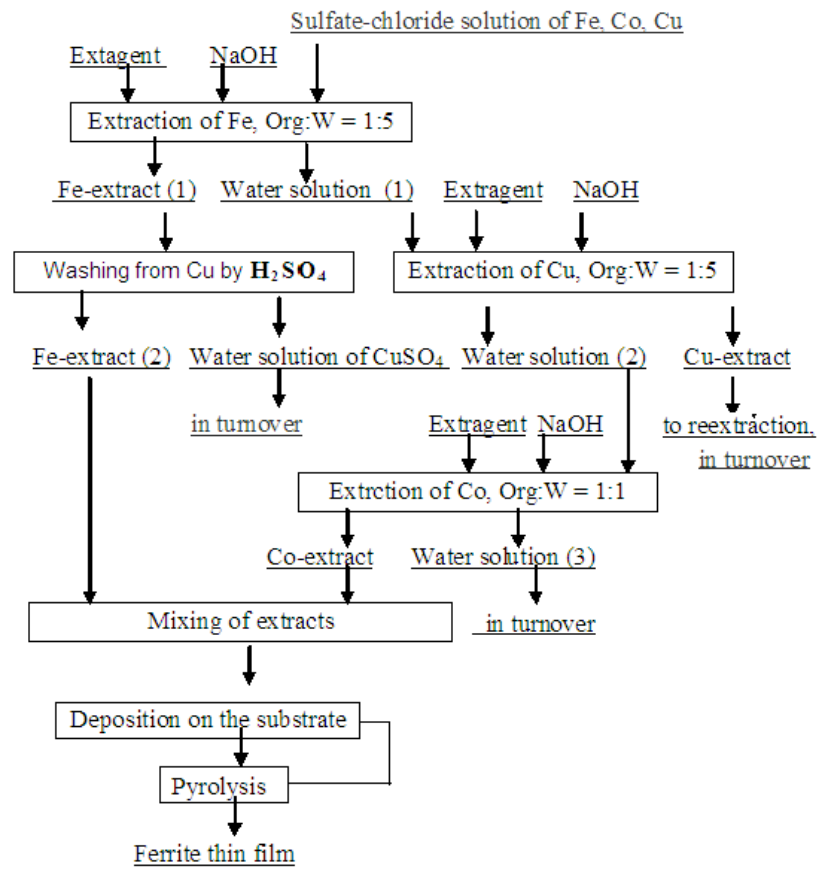

Figure 1. Technological scheme outlining manufacturing solution for producing cobalt-ferrite films

As a result, following complete extraction of iron the Fe extract (1) contains $21 \%$ of the copper. The cleaning of Fe-extract from the copper was performed by using $10 \%$ solution of $\mathrm{H}_{2} \mathrm{SO}_{4}$. On the second stage the extraction of copper from cobalt solution was repeated with extractant (VIC) and alkali addition. Cobalt has been extracted from water solution (2) after copper extraction. The resulting Fe and Co extracts do not contain impurities.

The efficiency of the extraction increases a counter current flow process is used.

The application of microwave heating for the Co-ferrite 
thin films thus obtained have same peculiarities in comparison with traditional induction heating. It is known that the high velocity of heating to $200 \%$ min promises the conservation of ultra fine structure of materials and allows to accelerate the processes of phase formation.

\subsection{Thermal Decomposition of Extracts}

The thermal decomposition of extracts in air atmosphere leads to the resultant simple and composite oxides. The thermal decomposition of extracts usually passes through 3 stages and concludes at $450-500{ }^{\circ} \mathrm{C}$.

Thermogravimetric study of extracts and their mixtures (Fig.2) demonstrates that water is removed at $90-150^{\circ} \mathrm{C}$, the excess of extractant is removed at $170-190^{\circ} \mathrm{C}$. Carboxylates decompose with removal of gases $\mathrm{CO} ; \mathrm{CO}, \mathrm{CH}_{4}, \mathrm{C}_{2} \mathrm{H}_{6}$ in the temperature range $250-470{ }^{\circ} \mathrm{C}$. By using gas-liquid chromatography (GLC) it was found that the $90 \% \mathrm{CO}_{2}, 8 \%$ $\mathrm{CO}$ and $2 \%$ of other gases are present in the gaseous product.

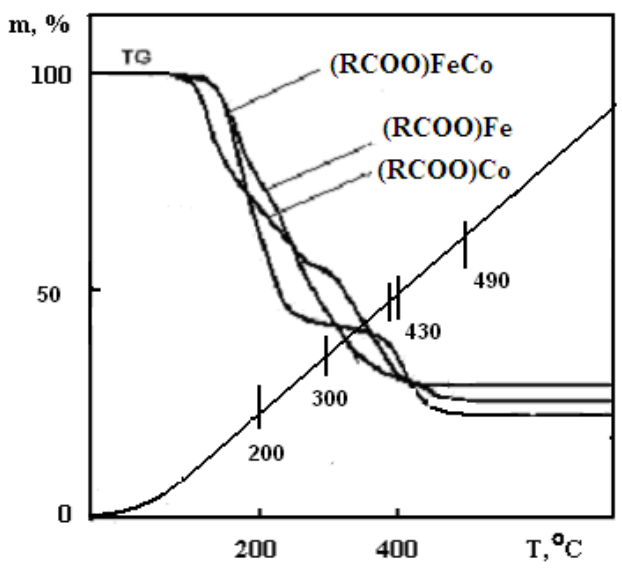

Figure 2. Termogravimetric curves of $\mathrm{Co}$ and $\mathrm{Fe}$ extracts

Metals concentrations of the cobalt and iron extracts were analyzed and mixed in a stoichiometric ratio $\mathrm{Fe}: \mathrm{Co}=2: 1$. The organic solution of extracts was deposited on glass by a spin coating technique and underwent pyrolysis with 5-10 times repetition in order to produce the films of cobalt ferrite. After 5-15 cycles of wetting and pyrolysis, the cobalt ferrite films were obtained with thicknesses of 100-500 nm. The as-deposited films were annealed at temperatures 350-650 ${ }^{\circ} \mathrm{C}$ during 1 hour in air.

It was found that the films became magnetic after pyrolysis at $450{ }^{\circ} \mathrm{C}$. X-ray diffraction (XRD) patterns of the films annealed at different temperatures are shown in Fig.3. The XRD pattern for a film annealed at $350{ }^{\circ} \mathrm{C}$ (see Fig.3, a) have a maximum at $20-22^{\circ} 2 \Theta$ indicating the existence of amorphous structure on the films.

With the increasing of the annealing temperature to $400-500{ }^{\circ} \mathrm{C}$ the crystallization occurred, as indicated by the appearance of the small peaks (Fig. 3, b) which was identified as a spinel structure. The broadening of the peaks testifies the presence of nanocrystalline grains in the film.
Particle size of about $18 \mathrm{~nm}$ was determined from the half-width of the X-ray diffraction peak using the Scherrer equation. This value is much lower than the grain size obtained for polycrystalline ferrite films prepared by the electron beam and magnetron deposition techniques $[7,8]$.

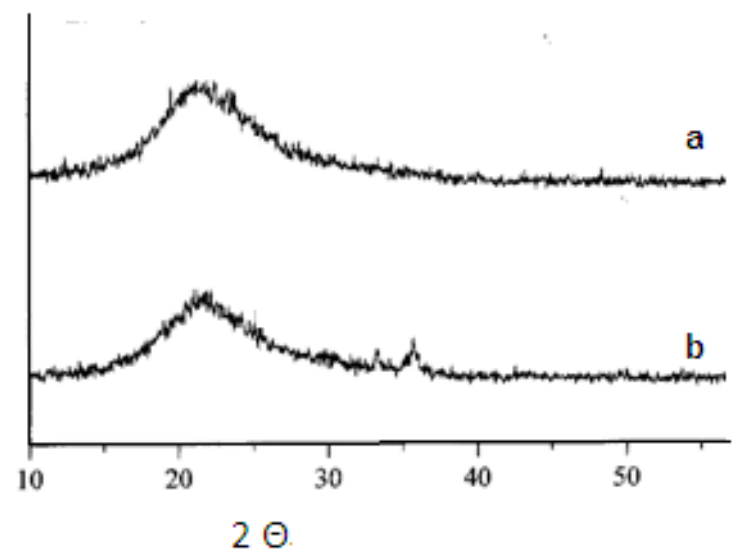

Figure 3. X-ray diffraction of $\mathrm{CoFe}_{2} \mathrm{O}_{4}$ films after annealing at $350{ }^{\circ} \mathrm{C}$ (a), $450{ }^{\circ} \mathrm{C}(\mathrm{b})$

$\mathrm{XRD}$ investigations demonstrated the crystalline phase of $\mathrm{FeCoO}_{2}$ thin films prepared by microwave heating of extracts deposited on glass substrates. The microphotographs from a scanning electron microscope (SEM) have shown the nanosize particles with dimension of about $10 \mathrm{~nm}$ (Fig. 4).

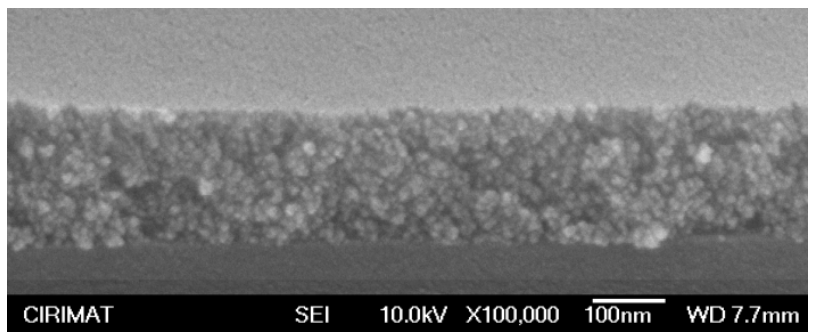

Figure 4. SEM image of $\mathrm{Fe}_{2} \mathrm{CoO}_{4}$ film prepared by microwavw pyrolysis of extracts solution

\subsection{Magnetic Properties of Co-ferrite Films}

The plot of magnetic in-plane and perpendicular plane hysteresis loops for the films was obtained via the technique of polar Kerr effect [9]. The typical magnetic loop curve of $\mathrm{CoFeO}_{4}$ with a perpendicular magnetic anisotropy is shown in Fig.5. This indicates the presence of the perpendicular magnetic anisotropy in cobalt ferrite films with composition $\mathrm{CoFe}_{2} \mathrm{O}_{4}$.

Ferrite films synthesized on quartz substrates by the extraction-pyrolysis technique have high values of the perpendicular magnetic anisotropy constant $\left(\mathrm{K}_{\mathrm{u}}=\right.$ $\left.0,8 \cdot 1,0 \cdot 10^{-5} \mathrm{~J} / \mathrm{m}^{3}\right)$, coercive force $\left(\mathrm{H}_{\mathrm{c}}=220-260 \mathrm{kA} / \mathrm{m}\right)$ and hysteresis squareness $(\mathrm{S}=0,8-0,9)$. Hysteresis squareness was calculate as $S=\theta_{\mathrm{k}} / \theta_{\mathrm{ks}}$, where $\theta_{\mathrm{k}}-$ magnitude of Kerr rotation in a field $H=0, \theta_{\mathrm{ks}}-$ in a field of saturation; $\theta_{\mathrm{F}}-$ magnitude of Faraday rotation. 


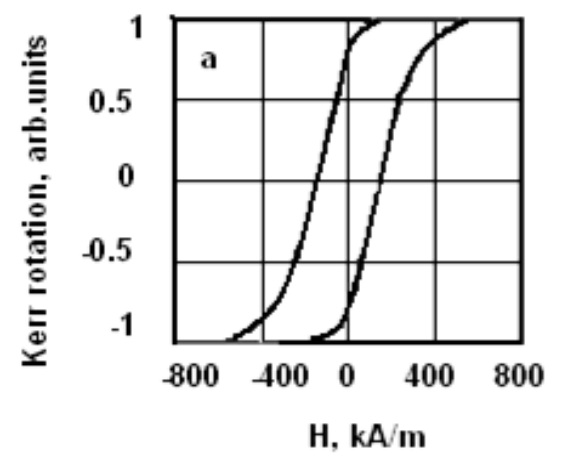

Figure 5. Magnetooptical hysteresis loops for $\mathrm{CoFe}_{2} \mathrm{O}_{4}$ film

The spectral dependence of the Faraday effect $\left(\theta_{\mathrm{F}}\right)$ of the $\mathrm{Co}_{0.6} \mathrm{Fe}_{2.4} \mathrm{O}_{4}$ film has two extremes (Fig. 6): $\theta_{\mathrm{F}}=2.5 \mathrm{deg} / \mu \mathrm{m}$ on $\lambda=0.5 \mu \mathrm{m}$ and $\theta_{\mathrm{F}}=-2.5 \mathrm{deg} / \mu \mathrm{m}$ on $\lambda=0.8 \mu \mathrm{m}$.

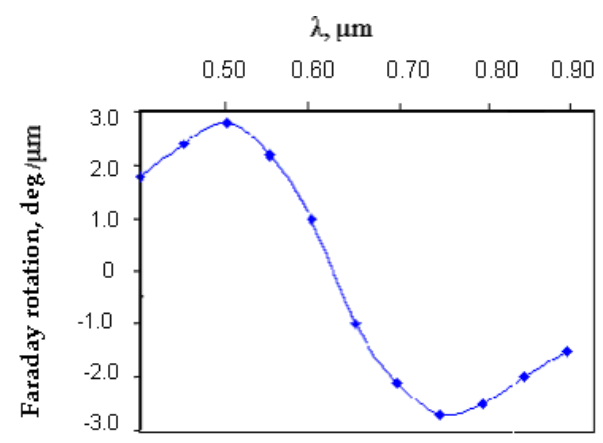

Figure 6. Spectral dependence of Faraday rotation on $\mathrm{CoFe}_{2} \mathrm{O}_{4}$ film

The value $\theta_{\mathrm{F}}$ on $\lambda=0.8 \mu \mathrm{m}$ is much higher than Faraday rotation on $0.8 \mu \mathrm{m}$ of other ferrite films, with the exception of Ce-substituted iron garnet films, but its magnetic properties make it the most suitable ferrite for the magneto-optical recording.

The practical interest presents the magnetooptical value $\Psi$ $=\mid 2 \theta_{\mathrm{F}} / \alpha$, where $\alpha$ - factor of an optical absorption, for wavelength 0.63 and 0.8 microns, that is equal to 0.5 and 3 degree respectively. These values are not lower than the values obtained for cobalt ferrite films by other method [10].

The magnetization of saturation for $\mathrm{CoFe}_{2} \mathrm{O}_{4}$ films obtained by microwave pyrolysis was evaluated as 100 Gs. Perpendicular magnetic anisotropy in films was not found, but nanosize films are characterized by the Kerr and Faraday effect. Therefore they are suitable for magneto optic applications.

It is apparent from the above results that the usage of an extractive-pyrolytic method allowed to obtain cobalt ferrite films with nanocrystalline structure having high values of magnetic and magnetooptical parameters. The values of the above-stated properties meet the requirements for magneto-optical applications.

\section{Conclusion}

By the extraction-pyrolysis technique the pure and homogeneous films have been derived from model of manufacturing solution. This technique is low cost and suitable for deposition of films on the large surfaces or on materials with complex forms. The structure of films deposited by the extraction-pyrolysis technique is an important factor for different functional applications. For instance, for obtaining films with perpendicular magnetic anisotropy and magneto-optic materials, the extractive-pyrolysis technique allows nanosize materials and materials with controllable size of grains to be derived.

\section{REFERENCES}

[1] Hongtao Cui, Wanzhong Ren, Wenhua Wang Synthesis on an ultra large scale of nearly monodispersed $\gamma-\mathrm{Fe}_{2} \mathrm{O}_{3}$ nanoparticles with $\mathrm{La}(\mathrm{III})$ doping through a sol-gel route assisted by propylene oxide // Journal of Sol-Gel Science and Technology. 2010, Vol. 54, Issue 1, 37-41

[2] Sanjoy C. Das, Robert J. Green, Jiban Podder, Tom Z. Regier, Gap Soo Chang Mixed-metal oxide nanopowders by liquid-feed flame spray pyrolysis (LF-FSP): Synthesis and processing of core-shell nanoparticles // The Journal of Physical Chemistry C . 2013, Vol.117, 12745-12753.

[3] T.N. Patrusheva, "Solution filming techniques", Krasnoyarsk: SFU, $301 \mathrm{p}$.

[4] M. Abe, M. Gomi, "Magneto-optical recording on garnet films”, J.Magn. Magn. Mater. Vol. 84, No.3, 1990, 222-228.

[5] A.I. Kholkin T.N. Patrusheva, "Extraction-pyrolysis technique for the oxide functional materials preparation", Moskow: Komkniga. 2006. 187 p.

[6] V.I. Kapustin, "High pure ultrafine oxide powders", Advanced materials. No. 5, 1998, 54-61.

[7] A.T. Ngo and M.P. Pileni, "Magnetic Fluids: Fabrication, Magnetic Properties, and Organization of Nanocrystals", New J. Phys., Vol. 4, 2002, 87.

[8] T. Itoh. Q. Zhang, M. Abe, Y. Tamaura, "Ferroelectrics", J. Appl. Phys. Vol.70, 1991, 6443.

[9] S.V. Vonsovsky, "Magnetizm", Moskow: Nauka, 1971.

[10] K.P.Polyakova, V. A Seredkin, V.F. Pavlov "Preparation and properties of polycrystalline ferrite films for magneto-optical memory", Sci. Appl. Photo.,Vol.40, No. 5, 391-396. 